I vana Regina Gonçalves ${ }^{1}$

CarlosPadovani ${ }^{2}$

Regina Célia Popim ${ }^{3}$
${ }^{1} \mathrm{H}$ ospital das Clínicas, Faculdade de M edicina de Botucatu, UNESP. Distrito deRubião J r s/n. Campus Universitário. 18618-970. Botucatu SP. I vana@fmb.unesp.br

${ }^{2}$ Departamento de

Bioestatística, Faculdade de

M edicina de Botucatu, UNESP.

${ }^{3}$ Departamento de Enfermagem, Faculdadede M edicina de Botucatu, UNESP.

\section{Caracterização epidemiológica e demográfica de homens com câncer de próstata}

\author{
Demographic and epidemiological characterization \\ of men with prostate cancer
}

Abstract This study aimed to identify the demographic and epidemiological characteristics of men with prostatecancer, assisted at theBotucatu School Hospital/U N ESP between January 1, 2000 and January 31, 2003. The data collected from 78 of 94 medical records included educational level, tobacco use, marital status, year of birth, diagnosis and symptoms. A quantitative method associated with descriptive statistics was used to this purpose. 50\% of the patients had not concluded elementary school, $27.39 \%$ werenon-smokers, $87 \%$ weremarried, $43 \%$ were aged $64-73$ years, $27.58 \%$ were diagnosed in 2000,63.2\% were retired, $20.7 \%$ showed weak urine stream as a symptom. Only $20 \%$ had undergone preventive exams and $77.9 \%$ had not completed elementary school. Prostate cancer accounted for $7.34 \%$ of the cancer cases, showing an incidence of $73,4 / 1000$. The study was conducted in this hospital for being a tertiary reference unit in the DIR XI ${ }^{1} .80 \%$ of the patients sought care when symptoms of the disease were already present. This makes us believe that the population still lacks information about prostate cancer, mainly as refers to incidence and prevalence related to age as a risk factor. This study will contribute to improving the care delivered to these patientsinduding early diagnosisand possiblecure, thus improving their quality of life.

Key words Cancer, Prostate, Characterization
Resumo 0 estudo pretende identificar características demográficas eepidemiológicas em homens com câncer de próstata, atendidos no H ospital das Clínicas da Faculdade de M edicina de Botucatu/ UNESP, entre 01/01/2000 e 31/01/2003. Foram colhidos dados em 78 de 94 prontuários, observando-se escolaridade, uso detabaco, estado civil, idade, diagnóstico e sintomatologia. Utilizou-se me todologia quantitativa associada à estatística descritiva. Foram encontrados $50 \%$ com $1^{\circ}$ grau incompleto, $27,39 \%$ não tabagistas, $87 \%$ casados, $43 \%$ entre 64 e 73 anos. Observou-se que 27,58\% foram diagnosticadosem 2000 , sendo $63,20 \%$ aposentados e 20,70\% com sintoma de jato urinário fraco. Apenas $20 \%$ tinham realizado exames preventivos e, desses, $77,90 \%$ possuíam $1^{\circ}$ grau incompleto. 0 estudo foi feito nesse hospital por ser considerado de referência terciária da Direção $R e-$ gional de Saúde de Botucatu -DIR XI'. Da amostra, 80\% procuraram o serviço apresentando sintomas, o que leva a crer que ainda haja falta de orientação da população quanto ao câncer depróstata, principalmente quanto à incidência e prevalência relacionadasà idade, como fator de risco. 0 estudo evidencia subsídios para um melhor direcionamento da assistência a esses pacientes, permitindo um diagnóstico precocee conseqüentepossibilidade de cura, além da melhora na qualidade devida.

Palavras-chave Câncer, Próstata, Caracterização 
Introdução

Atualmente, a Organização Mundial da Saúde considera o câncer como um problema de saúde pública. De acordo com dados divulgados por este órgão, há no mundo 10 milhões de pessoas com câncer e, se nenhuma alteração for feita, seremos 16 milhões de pessoas com câncer no ano de $2020^{2}$.

No Brasil, em 2003, tivemos 402.190 casos novos consolidados de câncer, sendo que os registros mostraram 126.960 óbitos decorrentes dessas patologias 3 . 0 câncer de próstata é considerado a segunda causa de óbito em homens adultos, sendo superado apenas pelo câncer de pulmão. Em nosso país, foram relatados 35.240 casos, sendo que a taxa de mortalidade para essa neoplasia no ano de 2003, de acordo com o Sistema de Informações de M ortalidade (SIM) , foi de 8.977 casos $^{4}$. Somente na região Sudeste, foram registrados 22.920 casos novos em 2003 e 4.370 foram a óbito, neste ano ${ }^{3}$. Para 2006, estimou-se a ocorrência de 472.050 casos novos de câncer no território nacional e de 47.280 casos novos de câncer de próstata ${ }^{5}$. 0 aumento observado nas taxas de incidência do câncer de próstata pode ser parcialmente justificado pela evolução dos métodos diagnósticos, pela melhoria na qualidade dos sistemas de informação do país e pelo aumento na expectativa de vida do brasileiro ${ }^{3}$.

Os principais fatores de risco descritos para o desenvolvimento do câncer de próstata são idade avançada, etnia e predisposição familiar. 0 envelhecimento é considerado o fator de risco maissignificante. A incidência do câncer de próstata em homens com idade superior a 50 anos é maior que $30 \%$, aumentando progressivamente até aproximadamente $80 \%$ aos 80 anos ${ }^{6}$. A incidência do câncer de próstata difere substancialmente entre os grupos étnicos. Assim éque afroamericanos têm incidências de dez a quarenta vezes maiores que os asiáticos ${ }^{7}$. A magnitude estimada do risco relativo em parentes de primeiro grau de afetados não parece diferir significativamente entre os grupos raciais (africanos, caucasianos e asiáticos) embora, como foi dito acima, ocorram grandes diferenças naincidência da doença entre estes grupos. Parentes de primeiro grau de pacientes com câncer de próstata apresentam risco aumentado de duas a três vezes, quando comparado a homens na população geral ${ }^{8,9}$. Entre outros fatores derisco, encontramse a dieta altamente calórica e os hormônios masculinos ${ }^{10}$.
O M inistério da Previdência Social ${ }^{11}$ salienta que uma parte significativa das neoplasias é atribuída a influências ambientais, particularmente àquelas relacionadas ao estilo de vida. Portanto, esta fração de ocorrência do câncer é potencialmente previnível. Alguns destes fatores agem nas fases iniciais deindução da carcinogênese, outros em fases subseqüentes como promotores e há também relato de casos que agem em ambas as fases. Assim, fatores relacionados à qualidade de vida influenciariam, significativamente, a ocorrência do câncer de próstata ${ }^{11}$.

A literatura refer $\mathrm{e}^{12}$ queo número de casos de diagnóstico de câncer de próstata entre os homens negros norte-americanos é de 79/100.000 enquanto que, considerando-seosjaponeses, que vivem no Japão, essa relação passa a ser de 4/ 100.000 homens. 0 estudo revela também que 0 índice é maior entre homens japoneses que migraram e vivem nos Estados Unidos da América. Tal constatação indica uma forte associação do diagnóstico a fatores ambientais e às condições de vida e alimentação do grupo ${ }^{12}$.

Dado o exposto, estima-se que um em cada dez homens, durante a vida, desenvolve carcinoma de próstata, clinicamenteevidente. Incidência maior ocorre em pessoas com idades mais avançadas e, também, predominantemente, entrehomens casados $^{13}$. A incidência é sempre subestimada porque muitos tumores permanecem assintomáticos durante toda a vida do indivíduo, sendo diagnosticados apenas em procedimentos de necropsia ${ }^{14}$.

A ocorrência histológica de uma lesão é um processo muito freqüente, relatado em aproximadamente um terço dos homens acima de 45 anos de idade. No entanto, a maioria dessas lesões não evolui para tumores clinicamente detectáveis ${ }^{15}$.

Estudos de necropsia revelaram que $30 \%$ dos homens acima de 50 anos que morreram por outras causas apresentam focos de adenocarcinoma na próstata. Esse número aumenta para $70 \%$ em homens acima de 80 anos de idade ${ }^{12}$.

Estudos sobre os sintomas evidenciam que, em geral, os pacientes com essa neoplasia têm descoberto o nódulo, por acaso, durante exames de rotina. Em outros métodos, é citado o achado incidental durante exames em decorrência do aumento da próstata. Já os não pal páveis seriam descobertos quando há uso da ultra-sonografia transretal durante exame físico, ou mediante estudo de hiperplasia prostática benigna. Afirmase que o câncer prostático localizado raramente causa sintomas, podendo camuflar outras doenças com sintomatologia de obstrução do trato 
de saída vesical, retenção urinária aguda, hematúria ou incontinência ${ }^{16}$.

Conhecer a fisiopatologia do câncer de próstata, o qual demora, em média, quinze anos para desenvolver-se até $1 c c^{3}$ de tamanho ${ }^{2}$, pode permitir esperança e, por outro lado, imprime responsabilidade para atuar nesse processo, em be nefício do paciente.

Diante do exposto, nos propusemos a esse estudo objetivando que ele possa oferecer subsídios no sentido de delimitar um perfil desses homens e, assim, se constituir em elemento para uma atenção especial e direcional na assistência aos pacientes, nos níveis primário e secundário de atenção à saúde. Ainda que não seja possível impedir o diagnóstico de câncer de próstata, pode-se realizá-lo precocementee, assim, iniciar o tratamento de forma a salvar vidas e impedir sofrimentos ainda maiores para estas pessoas e seus familiares.

\section{Objetivos}

Identificar as características demográficas e epidemiológicas dos pacientes quese submeteram a tratamento de câncer de próstata no Hospital das Clínicas da Faculdade de M edicina de Botucatu/U N ESP, no período de 01 dejanei ro de 2000 a 31 de janeiro de 2003, evidenciando características como faixa etária, estado civil, grau de alfabetização, história familiar da patologia, ano do diagnóstico e sintomatologia referida pelos acometidos. Traçar um perfil dessas características e, assim, obter subsídios para orientação da população que compõe grupo de risco, propondo formas de diagnóstico precoce e de prevenção.

\section{M aterial e método}

A coleta de dados foi realizada em prontuários médicos junto ao CIMED (Centro de Informática da M edicina) do HC da FMB/UNESP, nos meses de novembro e dezembro de 2003. Segundo o critério de inclusão, seriam examinados todos os prontuários de pacientes com diagnóstico de câncer de próstata atendidos no HC no período acima citado, utilizando-se a metodologia quantitativa, que considera o tamanho do objeto da pesquisa e sua numeração associada à estatística descritiva ${ }^{17}$.

\section{Resultados}

H avia 94 prontuários que atendiam ao critério de inclusão. Considerando-se que, até o final da coleta, alguns não se encontravam disponíveis, foram consultados 78 prontuários.

Distribuição do câncer de próstata segundo o grau de escolaridade

Dividindo-seos pacientes em grupos deacordo com a alfabetização, vê-se que, dos 78 prontuários examinados, $50 \%$ possuíam $1^{\circ}$ grau incompleto, $28,57 \%$ estavam cadastrados como alfabetizados, $7,14 \%$ possuíam $1^{\circ}$ grau completo, $3,58 \%$ apresentavam $3^{\circ}$ grau completo, $3,57 \%$ possuíam $2^{\circ}$ grau incompleto, $2,38 \%$ ○ $2^{\circ}$ grau completo efoi registrado percentil nulo para pacientes com $3^{\circ}$ grau incompleto. Em relação aos analfabetos, o total somou 3,57\%. N ão constava a informação sobre o grau de escolaridade em $1,19 \%$ dos pacientes.

Distribuição do câncer de próstata segundo o uso de tabaco pelos pacientes

Os prontuários que continham esta informação totalizaram $47,72 \%$ da amostra. Desses, $27 \%$ dos pacientes não faziam uso do tabaco, $11,9 \%$ faziam e, aproximadamente, 8,33\% eram ex-fumantes. Houve uma predominância de homens não tabagistas. M erece destaque 0 fato de que $52,38 \%$ dos prontuários não explicitavam a tão relevante informação sobre o uso de tabaco, nesses pacientes com diagnóstico de câncer.

Distribuição do câncer de próstata segundo o estado civil dos pacientes

Verificou-se que $80 \%$ dos homens avaliados eram casados, $8,23 \%$, viúvos, $4,7 \%$, solteiros, $4,7 \%$, unidos consensual mente, $2,37 \%$, divorciados.

Distribuição do câncer de próstata segundo a idade dos pacientes

Observou-se maior concentração de pacientes na faixa de 69 a 73 anos, representando $45 \%$ da mostra, e 23,57\% de 63 a 68 anos de idade. Os demais somam 13,95\%, com 79 a 84 anos; $20 \%$, de 74 a 78 anos, $7,09 \%$ de 59 a 63 anos, 2,37\% de 54 a 58 anos e $3,57 \%$ de 49 a 53 anos de idade. 
Distribuição do câncer de próstata segundo o ano do diagnóstico

Verificou-se que a maior concentração dos diagnósticos realizados ocorreu no ano de 2000, abrangendo $27,58 \%$ dos pacientes, seguido do ano 2002 , com $22,98 \%$ e de 2001 , com $22,58 \%$. Os restantes foram diagnosticados em 1999, com 3,48\%. Em 1997, 1998, 2003, somaram 2,29\% e, em 1991, com 1,14\%. Cabe salientar que em 8,04\% dos prontuários não havia informações a esse respeito.

Distribuição do câncer de próstata segundo atividade

Verificou-se que a maioria dos pacientes, ou seja, $63,2 \%$, era constituída por aposentados. Outras atividades exercidas foram: Iavrador $(4 \%)$, comerciante $(8 \%)$, motorista $(4,45 \%)$. As demais, contando cada uma com $1,15 \%$, foram: jardineiro, vendedor, avicultor, gerente de produção, dentista, britador, farmacêutico, supervisor de ensino, agropecuarista, faqueiro, re presentante comercial, alfaiate e desempregado.
Esta análise poderia ser mais consistente se os prontuários informassem qual foi a profissão exercida por estes aposentados, pois tal associação poderia contribuir para consolidar e/ou substanciar fatores de risco.

Distribuição do câncer de próstata segundo a sintomatologia

A sintomatologia relatada pelos pacientes encontra-se expressa no Q uadro 1.

Verificou-se que $15 \%$ dos pacientes realizaram exames preventivos, sendo que $62,5 \%$ deles em 2001, 25\% em 2000 e 12,5\%, em 2002.

Distribuição do câncer de próstata segundo a realização de exames preventivos e graus de escolaridade

Verificou-se que, dos pacientes que realizaram exames preventivos, $77,9 \%$ têm 10 grau incompleto, $11,11 \%, 1^{\circ}$ grau completo e $6,55 \%$, 3ㅇ grau completo. Os analfabetos somaram 5,55\% cada um. Isso indica que a maioria deles possuía baixo nível de alfabetização.

Quadro 1. Distribuição do câncer de próstata segundo a sintomatologia.

\begin{tabular}{|c|c|}
\hline $\begin{array}{l}\text { Freqüência relativa } \\
\text { a cada sintoma (\%) }\end{array}$ & Sintomatologia \\
\hline 20,7 & Jato urinário fraco \\
\hline 17,3 & Nictúria \\
\hline 15 & Exames de rotina/sem sintomas \\
\hline 10,3 & Disúria \\
\hline 6,7 & Dor à micção; dificuldade ao urinar \\
\hline 5,7 & Perda urinária \\
\hline 5 & Polaciúria \\
\hline 4,5 & Poliúria; ardência miccional; retenção urinária;achado laboratorial \\
\hline 3,5 & Dificuldade para iniciar a micção \\
\hline 2,3 & Dor no escroto; hematúria; perda de peso; verrugas no pênis \\
\hline 1,14 & $\begin{array}{l}\text { Dor em região lombar; dor supra pélvica; dor nas pernas ; dor no ventre; dor na região } \\
\text { cervical; dor em fossa ilíaca; dor ao urinar; gotejamento terminal; obstrução urinária; jato } \\
\text { demorado; crescimento lento e progressivo do testículo; oligúria; urgência miccional; } \\
\text { diminuição do tempo deereção; baixadafreqüência miccional; intestino preso; evacuação } \\
\text { com sangue; sensação de esvaziamento vesical; hesitação urinária) }\end{array}$ \\
\hline
\end{tabular}




\section{Discussão econclusão}

A incidência ea prevalência do câncer de próstata em nosso país motivaram a busca do perfil dos homens acometidos pelo câncer de próstata atendidos no HC da Faculdade de Medicina da UNESP de Botucatu. Para tanto, utilizou-seuma metodologia que permitisse uma busca retrospectiva, de maneira a tornar possível descrever e relacionar aspectos epidemiológicos e demográficos nessa população.

0 estudo foi fundamentado no fato deste hospital ser referência terciária da Direção Regional de Saúde de Botucatu - DIR XI. Ela está localizada à Sudoeste do estado de São Paulo, tem sede em Botucatu, conta com 532.761 habitantes ecompreendetrinta municípios ${ }^{18}$. A procura dos doentes por esta instituição é motivada pela confiança na assistência prestada e também na incapacidade que o município de origem dos mesmos tem em proporcionar atendimento similar ${ }^{1}$. 0 serviço prestado pelo hospital é importante e os pacientes atendidos com câncer de próstata constituem-se em amostra regional. Esse aspecto faz com que o estudo e a investigação dos parâmetros anteriormente apresentados assumam importância maior.

Esta investigação revelou que, dos homens atendidos no triênio de 2001-2003, 7,34\% apresentam diagnóstico de câncer de próstata em re lação aos demais cânceres, uma incidência de 73,4/ 1.000 na população acometida. A análise dos 78 prontuários, de um total de 94 , foi realizada no sentido de melhor caracterização desses homens e na busca de fatores de risco associados ao câncer de próstata. Nesse sentido, o perfil dos pacientes atendidos revelou predominância da cor branca (95\%), e faixa etária entre 64 e 73 anos, 0 que corrobora os dados da literatura, que apontam que o câncer de próstata tem ocorrência em um terço dos homens acima de 45 anos, na população ocidental ${ }^{19}$. Valeenfatizar que 0 aumento da incidência na população é também uma de corrência do aumento da expectativa de vida dos brasileiros verificado ao longo desse século, cuja tendênciaéultrapassar os 70 anos no ano de2020, segundo o M inistério da Previdência Social ${ }^{11}$.

No que se refere ao grau de escolaridade, é possível observar que esses homens apresentam, em geral, baixa escolaridade; porém, em sua maioria, revelam algum grau de instrução, sendo que $49 \%$ têm $1^{\circ}$ grau incompleto.

Quanto ao uso de tabaco, a pesquisa contou com $11,9 \%$ constando como fumantes. No entanto, 52,38\% dos prontuários não continham esta informação, fato este causador de estranheza por serem dados colhidos em um hospital escola e estar o hábito de fumar associado com a morte precoce em uma estimativa anual de 80 mil pessoas, número que vem aumentando todos os anos ${ }^{19}$. A maioria dos pacientes era casada e mais da metade relatavam ser aposentados. Considerando que há o direito ao benefício de aposentadoria assegurado, por lei, para homens com mais de 65 anos, observa-se que há coerência, já que a maioria dos homens pesquisados, por meio dos prontuários, apresentavam idade entre 64 a 73 anos ${ }^{11}$.

Dos prontuários acessados, ainda pode-se evidenciar que apenas $15 \%$ dos pacientes foram diagnosticados em exame de rotina, sem apresentar sintomatologia. Os demais apresentavam algum tipo de sintoma, revelando que a busca pelo serviço foi em função da presença da doença já instalada. O s sintomas mais freqüentes relatados concordam com a literatura, e foram: o hábito de levantar várias vezes à noite para urinar, dificuldadeno ato miccional edor durantea micção ${ }^{20}$. I mportante lembrar que polaciúria e sensação de esvaziamento incompleto também foram relatados ${ }^{14}$.

Entre as pessoas que realizaram exame de rotina, um terço o fizeram em 2001. Alguns prontuários traziam anotações referentes à "campanha", sugerindo, então, que houve campanha de prevenção nesse ano. No entanto, consultando a Secretaria M unicipal de Saúde, verifica-se que a campanha realizada na cidade foi a de prevenção de câncer mamário, o que leva a inferir que isto podeter despertado um interesse maior pelo câncer singular nos homens, o de próstata.

Ao acessar os prontuários médicos, observasequehá uma carência de informações precisase relevantes acerca do diagnóstico confirmado. No caso do câncer de próstata, faltaram informações sobre história familiar da doença, uso do tabaco, ocupação anterior à aposentadoria. Tais dados incompletos prejudicaram uma análise conclusiva.

Esses registros indicam que os pacientes, em sua maioria, procuraram o serviço quando já havia presença de sintomas. Isso sinaliza para uma falta de orientação da população masculina a respeito do câncer de próstata, em relação à incidência, prevalência eidade, enquanto fatores de risco. Não há informação no prontuário sobre a dificuldade ou não de acesso ao serviço de saúde especializado, o que também pode ser um fato de busca tardia para o diagnóstico do câncer de próstata. 
Embora este estudo não tivesse o objetivo de enfocar o tratamento do câncer de próstata, é notório o conceito de que, quando se faz um diagnóstico precoce e a prevenção propriamente dita, há chance maior desucesso na luta contra o câncer a médio e longo prazo.

\section{Colaboradores}

IR Gonçalves, RC Popim e CR Padovani participaram igualmente das etapas da elaboração do artigo.

\section{Referências}

1. M endes HWB. Regionalização da assistência à saúde: análise da demanda ao serviço de urgência/emergência de um hospital universitário [tese]. São Paulo (SP): Universidade de São Paulo; 2003.

2. Organização M undial da Saúde. [site da Internet]. 2003 [acessado 2003 Nov 13]. Disponível em: http/ /www.who.int/cancer/em/

3. Instituto Nacional do Câncer. Estimativas 2003. [site da Internet]. 2003 [acessado 2003 Nov 13]. Disponível em: http://www.inca.gov.br/estimativas/2003

4. Brasil. Ministério da Saúde. Indicadores de mortalidade. [site da Internet]. 2003 [acessado 2006 M aio 12]. Disponível em: http://tabnet.datasus.gov.br/cgi/ tabcgi.exe?idb2006/c10.def.

5. Instituto Nacional do Câncer. Estimativas 2006. [site da Internet]. 2006 [acessado 2007 Jun 06]. Disponível em: http://www.inca.gov.br/estimativas/2006

6. Foster CS, Ke Y. Steam cells in prostatic epithelia. Rev Int J Pathol 1997; 78:311-329.

7. H sing AW, Tsao L, Devesa SS. International trends and patterns of prostate cancer incidence and mortality. Rev Int J Cancer 2000; 85:60-67.

8. Carter BS, Carter HB, Isaacs JT. Epidemiologic evidence regarding predisposing factors to prostate cancer. Prostate 1990; 16:187-197.

9. Goldgar DE, Easton DF, Cannon-Albright LA, Skolnick MH. Systematic population-based assessment of cancer risk in first-degree relatives of cancer probands. Rev J Natl Cancer Inst 1994; 86:1600-1608.

10. Epstein JI, Amin M, Boccon-Gibod L, Egevad L, Humphrey PA, Mikuz G, Newling D, Nilsson S, Sakr W, Srigley JR, Wheeler TM, Montironi R. Prognostic factors and reporting of prostate carcinoma in radical prostatectomy and pelvic lymphadenectomy specimens. Rev Scand J U rol Nephrol Suppl 2005; 216:34-63.

11. M inistério da Previdência Social. Aposentadoria por idade. [site da Internet]. 2003 [acessado 15 N ov 2003]. Disponível em: http://www.previdenciasocial.gov. br/02_01_07.asp
12. Barrios $\mathrm{CH}$. Câncer de próstata. In: Murad AM, Katz A, organizadores. Oncologia, bases clínicas do tratamento. Rio de Janeiro: Guanabara Koogan; 1996. p. $220-227$.

13. Ferreira V, Nardi AC. Câncer de próstata. In: Netto JR NR, organizador. Urologia prática. São Paulo: Atheneu; 1999. p.237-246.

14. Reyes BL, Hanhan IWF. Câncer da próstata. In: Borden EC, Bosh FX, Campbell RJ, Coleman MP, Drinkwater NR, Eckhart $S$, organizadores. $M$ anual de oncologia clínica. São Paulo: Fundação Oncocentro de São Paulo; 1997. p. 311-316.

15. Conforti-Froes NTD, Louro ID, Lima JO. Câncer de próstata. In: Louro ID, Llerena JC, Melo MSVD, Ashton-Prolla P, Conforti-Froes N, organizadores. Genética molecular do câncer. São Paulo: M.S.G Produção Editorial; 2002. p. 223.

16. Narayan P. Neoplasias da próstata. In: Smith DR, organizador. U rologia geral. Rio de Janeiro: Guanabara Koogan; 1994. p. 292-314; 305-306.

17. Campana AO, Padovani CA, Iaria CT, Freitas CBD, Paiva SAR, Hossne WS. Investigação científica na área médica. São Paulo: Manole; 2001. p.52-94.

18. Sistema Estadual de Análise de Dados. Fundação Sistema Estadual de Análise de Dados. Informações dos municípios paulista. São Paulo, 2003. [site da Internet] [acessado $2007 \mathrm{M}$ ai 31]. Disponível em: http://www.seade.gov.br

19. Souto CAV. Tratamento do câncer de Próstata. In: Schwatsmann G, organizador. Oncologia clínica. Porto Alegre: Artes M édicas; 1991. p. 399-406.

20. UNICAM P. Núcleo de Informática Biomédica. [site da Internet]. 2006 [acessado 2006 M ai 12]. Disponível em: http//www.nib.unicamp.br

Artigo apresentado em 03/05/2006

Aprovado 03/05/2007

Versão final apresentada em 01/04/2008 